\title{
PARTICLE SIMULATIONS OF DARHT-II TRANSPORT SYSTEM
}

\author{
Brian R. Poole, Yu-Jiuan Chen, LLNL, Livermore, CA 94550, USA
}

\begin{abstract}
The DARHT-II beam line utilizes a fast stripline kicker to temporally chop a high current electron beam from a single induction LINAC and deliver multiple temporal electron beam pulses to an x-ray converter target. High beam quality needs to be maintained throughout the transport line from the end of the accelerator through the final focus lens to the $\mathrm{x}$-ray converter target to produce a high quality radiographic image. Issues that will affect beam quality such as spot size and emittance at the converter target include dynamic effects associated with the stripline kicker as well as emittance growth due to the nonlinear forces associated with the kicker and various focusing elements in the transport line. In addition, dynamic effects associated with transverse resistive wall instability as well as gas focusing will affect the beam transport. A particle-in-cell code is utilized to evaluate beam transport in the downstream transport line in DARHT-II. External focusing forces are included utilizing either analytic expressions or field maps. Models for wakefields from the beam kicker, transverse resistive wall instability, and gas focusing are included in the simulation to provide a more complete picture of beam transport in DARHT-II. From these simulations, for various initial beam loads based on expected accelerator performance the temporally integrated target spot size and emittance can be estimated.
\end{abstract}

\section{INTRODUCTION}

High-resolution $\mathrm{x}$-ray radiography on the $2^{\text {nd }}$ axis of the Dual Axis Radiographic Hydrodynamic Test Facility (DARHT-II) requires that a $2 \mathrm{kA}, 18.4 \mathrm{MeV}$ electron beam be focused to a small $(\sim 1 \mathrm{~mm})$ time integrated spot on an X-ray converter target. The DARHT-II beam line has been discussed previously [1] and is shown in Fig. 1. Four electron beam pulses are extracted from the $2 \mu \mathrm{s}$ long pulse from the accelerator using a fast kicker system [2]. The kicker system directs the beam into either a beam dump or the target beam line. Normally, a static bias dipole magnet is used to direct the beam into the dump beam line. The quadrupole septum magnet gives an additional dipole kick to the off-axis beam to sufficiently bend the beam into the dump. When the fast stripline kicker is energized, the effective kick from the bias dipole is cancelled by the dynamic dipole field associated with the kicker, and the beam is injected into the center of the quadrupole septum magnet where the beam experiences a defocusing force in the kick plane. A set of four Collins' quadrupoles re-establishes a round beam after the quadrupole septum magnet after which the beam proceeds to the $\mathrm{X}$-ray converter target through a solenoid and the final focus solenoid. Initially, the TRANSPORT code is utilized to construct the appropriate magnetic transport system. A typical TRANSPORT simulation, shown in Fig. 2 shows the beam envelope from the accelerator exit to the x-ray converter target. Particle simulations provide additional information on the performance of the beam line. For example, nonlinear forces due to higher order multipoles, nonlinear space charge and image forces can provide information on emittance growth in the beam line. Time dynamic effects, due to kicker wakefields are also incorporated into simulations. Beam halo and particle loss can also be examined using particle simulations. A K-V particle load based on the $\sigma$-matrix from a

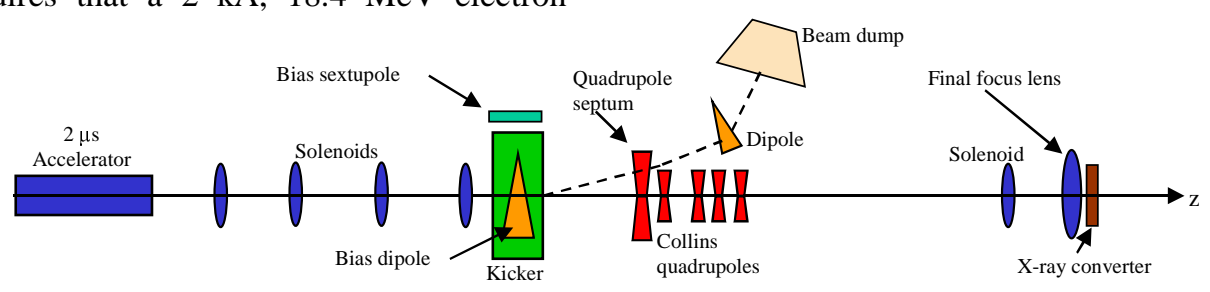

Figure 1: DARHT-II beam transport line.

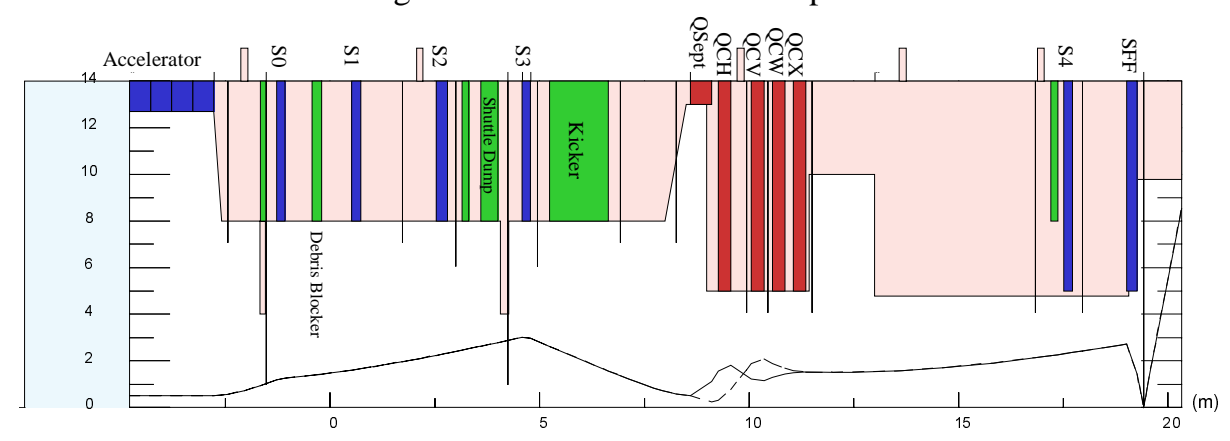

Figure 2: Transport simulation showing DARHT-II beam envelope from accelerator exit to x-ray converter target. 
TRANSPORT simulation is used for the initial beam conditions, but more complex beam loads may be used from the results of other simulation codes.

\section{MODELLING}

Multi-slice simulations are used to model the transport of a long pulse beam from the accelerator exit, through the kicker system, and to the x-ray converter target. Analytic field distributions are typically used to describe the various magnetic lenses and kicker along the transport line. However, the kicker deserves a more detailed description. The stripline kicker, shown schematically in Fig. 3 is designed to spatially separate an electron beam into two separate beam lines.
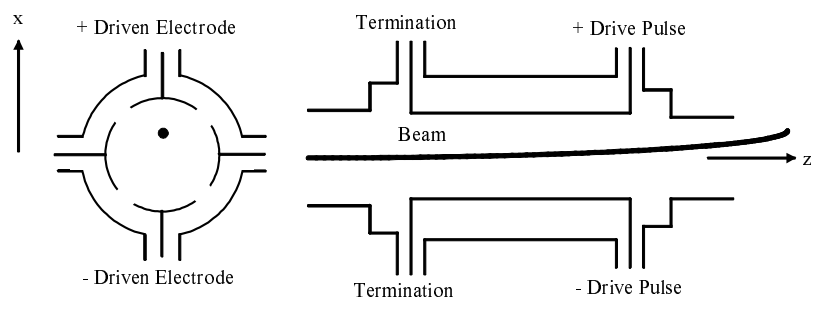

Figure 3: Schematic of fast beam kicker.

A voltage pulse is applied to the downstream ports of the kicker and the beam is kicked by the electric and magnetic fields associated with the TEM waves propagating on the strip transmission lines. The potential within the kicker plates, $(r<b)$ is given by Equation 1

$$
V=\frac{4 V_{p}}{\pi} \sum_{n=o d d}\left(\frac{1}{n}\right) \sin \left(\frac{n \theta_{0}}{2}\right) \cos (n \theta)\left(\frac{r}{b}\right)^{n}
$$

where $b$ is the interior radius of the kicker plates, $\theta_{0}$ is the angle subtended by each kicker plate, and $V_{p}$ is the kicker plate voltage. The $n=1$ term represents the transverse dipole force that provides the beam steering and terms with $n \geq 3$ will contribute to the emittance growth in the structure. The kicker system also includes a static bias sextupole winding to cancel the inherent sextupole field component associated with the dynamic kicker fields. The sextupole bias field is required to minimize emittance growth in the kicker system for most of the duration of the switched beam.

Particle-in-cell (PIC) simulations for the DARHT-II beam line incorporate beam corkscrew and energy variation in the beam initial conditions at the entrance to the beam line. Space charge, image forces, and charge and current redistribution in the particle slices as well as tallying particle loss along the beam line are included in the simulations. Figure 4 shows an initial particle load of 2000 particles for a single slice of a nominal $18.4 \mathrm{MeV}$ $\mathrm{K}-\mathrm{V}$ beam with an un-normalized emittance of 3.25 $\mathrm{cm}-\mathrm{mr}$ at the accelerator exit. Figure 5 shows the result of single slice transport of the beam slice to the target.
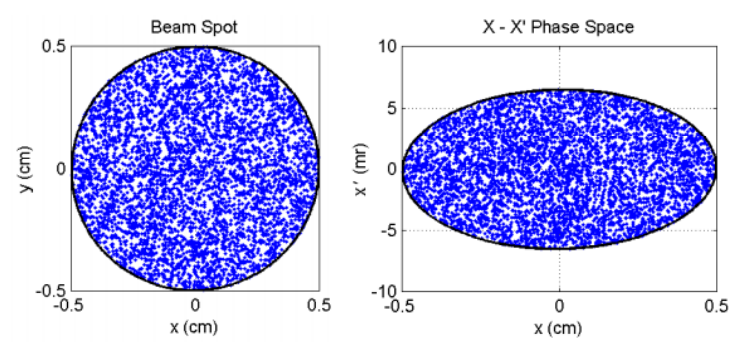

Figure 4: Particle load at accelerator exit
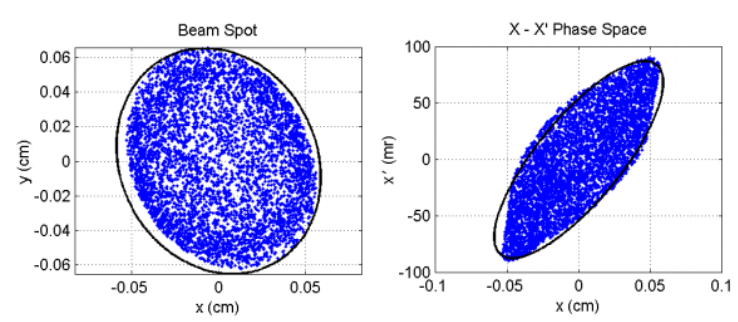

Figure 5: Beam spot and phase space near target plane.

A voltage pulse $V_{p}(t)$ is applied to the kicker resulting in a temporal sweep of the beam centroid and angle at he kicker exit. Figure 6 shows the beam envelope projection in the kick plane during the transient phase of the kicker switch and at steady state.
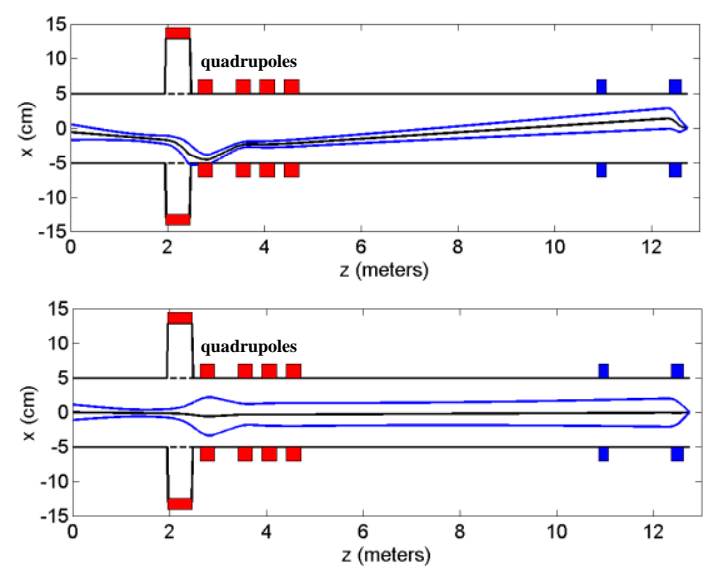

Figure 6: Beam envelopes during the kicker switch phase and at steady state. The kicker exit is at $\mathrm{z}=0$.

An important figure of merit in the performance of the DARHT-II beam line is the time integrated spot size on the $x$-ray converter target. Figure 7 shows a cumulative or time-integrated phase space at the $\mathrm{x}$-ray converter target allowing a bounding ellipse to provide an effective time integrated spot size and emittance. The effective spot size is $1.34 \mathrm{~mm}$ in $x$ and $1.7 \mathrm{~mm}$ in $y$ and the effective emittances are $3.5 \mathrm{~cm}-\mathrm{mr}$ and $5.4 \mathrm{~cm}-\mathrm{mr}$ in $x$ and $y$ respectively. 

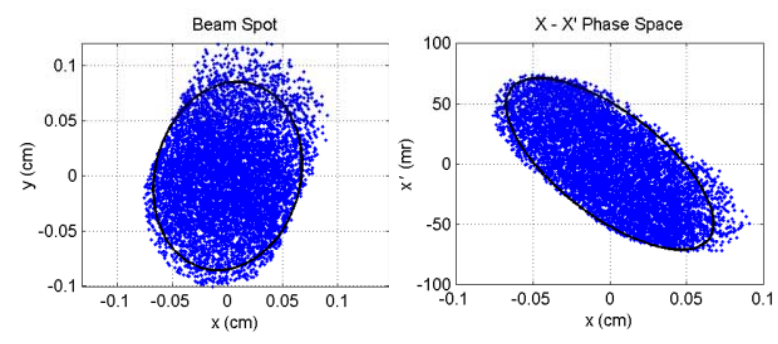

Figure 7: Time integrated target spot and phase space.

To quantify the spot size for non-uniform and non-circular beam spots a more useful definition of spot size is the $50 \%$ modulation transfer function (MTF) equivalent spot size. The modulation transfer function is defined as

$$
F_{\theta}(k)=\int_{0}^{\infty} n_{\theta}(r) J_{0}(k r) r d r
$$

and

$$
\operatorname{MTF}_{\theta}(k)=\left|\frac{F_{\theta}(k)}{F_{\theta}(0)}\right|
$$

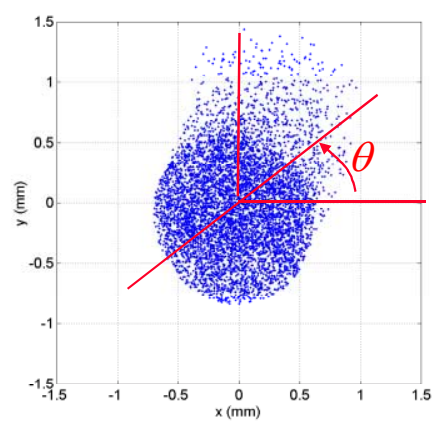

Figure 8: Time integrated spot profile.

The 50\% MTF value is defined from the value of $k$ when the MTF is $50 \%$ of its value at $k=0$. This value of $k$ is denoted as $k_{.5}$.The equivalent spot radius is defined as the radius of a uniform circular spot that gives the same value of $k_{5}$.The MTF for a uniform circular spot can be evaluated exactly as

$$
\operatorname{MTF}(k)=2\left|\frac{J_{1}(k a)}{(k a)}\right|
$$

where $a$ is the radius of the uniform spot. The 50\% MTF for a uniform spot occurs at $k_{5} a=2.215$. Therefore the MTF spot radius is given by

$$
a_{\mathrm{MTF}}=\frac{2.215}{k_{.5}}
$$

Applying this definition to the target spot shown in Fig. 7 allows the spot size to be calculated for as a function of angle, $\theta$. Figure 9 shows the angular variation of the spot shown in Fig. 7. Figure 9 shows an average spot diameter of $1.48 \mathrm{~mm}$. The transverse beam motion caused by the switching process associated with the kicker is the primary cause of the beam ellipticity.

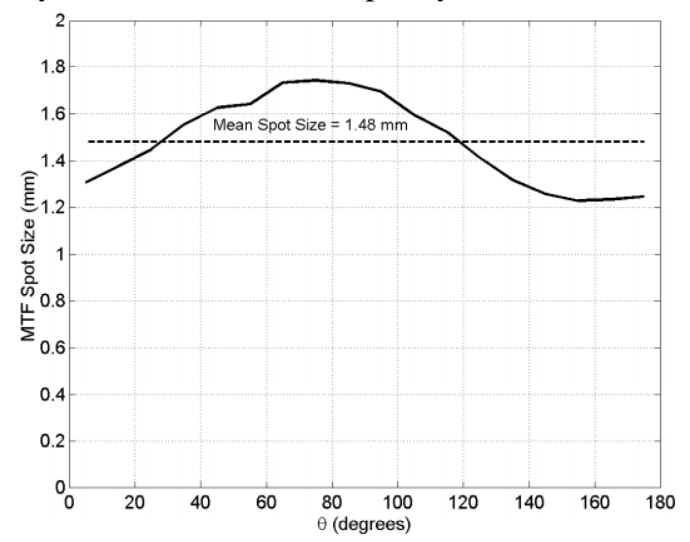

Figure 9: MTF spot size on target.

\section{CONCLUSIONS}

Multi-slice particle simulations provide detailed information about beam line performance based on a variety of physics models included in the simulations code. The time-integrated spot size is primarily determined by the dynamics associated with the kicker switch, as is the spot ellipticity. Additional physics models are being incorporated in the MULTISLICE code to include gas focusing, time dynamic effects such as ionhose instability, and transverse resistive wall instability.

\section{ACKNOWLEDGEMENTS}

This work was performed under the auspices of the U.S. Department of Energy by University of California Lawrence Livermore National Laboratory under contract No. W-7405-Eng-48.

\section{REFERENCES}

[1] A. C. Paul, G. J. Caporaso, Y. -J. Chen, Y. J. (Judy) Chen, G. Westenskow, "The Beamline for the Second Axis of the Dual Axis Radiographic Hydrodynamic Test Facility," PAC'99, New York, March 1999.

[2] B. R. Poole, G. J. Caporaso, Y. J. (Judy) Chen, L. -F. Wang, "Analysis and Modeling of a Stripline Beam Kicker and Septum," LINAC'98, Chicago, August 1998. 